\title{
A study of 17 cases for the identification of prognostic factors for anaplastic thyroid carcinoma
}

\author{
TAKASHI MASUI ${ }^{1}$, HIROKAZU UEMURA $^{1}$, ICHIRO OTA $^{1}$, TAKAHIRO KIMURA ${ }^{1}$, \\ DAISUKE NISHIKAWA ${ }^{2}$, TOSHIAKI YAMANAKA ${ }^{1}$, KATSUNARI YANE $^{2}$ and TADASHI KITAHARA ${ }^{1}$ \\ ${ }^{1}$ Department of Otolaryngology-Head and Neck Surgery, Nara Medical University, Kashihara, Nara 634-8522; \\ ${ }^{2}$ Department of Otolaryngology, Kinki University School of Medicine, Nara Hospital, Ikoma, Nara 630-0293, Japan
}

Received May 1, 2020; Accepted September 7, 2020

DOI: $10.3892 /$ mco.2020.2163

\begin{abstract}
Anaplastic thyroid cancer (ATC) has a poor prognosis. ATC accounts for only $1-2 \%$ of all thyroid carcinomas, yet it is one of the most lethal neoplasms in humans. Notably, there are no established treatment protocols for ATC. The present study investigated the prognostic and predictive factors of ATC. A retrospective analysis was conducted on 17 patients with histologically confirmed ATC. The median overall survival of all patients was 3.8 months. In patients under the age of 70 years, the statistically significant prognostic factors indicating longer survival were the absence of distant metastasis and treatment by radical resection. Furthermore, in contrast to previous findings, tumor size and white blood cell count were not associated with ATC prognosis in the present cohort. Importantly, tracheostomy did not contribute to improvement of prognosis and should perhaps not be considered, when unnecessary, to preserve the patient's quality of life. Prognostic factors for ATC are critical to clinicians to enable them to determine which patients will benefit from aggressive treatment strategies, as opposed to supportive care.
\end{abstract}

\section{Introduction}

Anaplastic thyroid cancer (ATC) accounts for $1-2 \%$ of all thyroid cancers, but has a very poor prognosis $(1,2)$. It is an aggressive disease with a mean survival time of 3-6 months after initial diagnosis, and a one-year survival prognosis of $5-20 \%$ (3).

The poor prognosis of ATC results from a tumor biology that promotes aggressive tumor growth with rapid invasion of the surrounding structures of the thyroid gland, including muscles, trachea, esophagus and recurrent laryngeal nerve,

Correspondence to: Dr Takashi Masui, Department of Otolaryngology-Head and Neck Surgery, Nara Medical University, 840 Shijo-cho, Kashihara, Nara 634-8522, Japan

E-mail: masui@naramed-u.ac.jp

Key words: anaplastic thyroid carcinoma, prognostic factor, prognostic index in addition to the development of distant metastasis $(4,5)$. Unfortunately, no established treatment strategies exist for ATC. Although radical resection of the tumor is a common therapeutic option, previous studies suggest that concurrent postoperative radiotherapy and chemotherapy may offer further survival benefits $(6,7)$. However, these treatments do not greatly improve the prognosis of ATC.

Importantly, there are some patients who have had long-term survival. In these patients, improved prognosis was related to treatment by radical resection. Currently, reported prognostic factors for ATC include acute symptoms, tumor size $>50 \mathrm{~mm}$, presence of distant metastasis, WBC count of over $10,000 / \mathrm{mm}^{2}, \mathrm{~T} 4 \mathrm{~b}$ and age over 70 (3). However, these reports are few and in between, thus, we investigated the key prognostic factors for ATC in 17 patients of our own.

\section{Materials and methods}

Background. Seventeen patients with histologically confirmed ATC treated between 1990 and 2019, were identified from the thyroid databases of the Nara Medical University in Japan. The inclusion criteria was evidence of ATC at histopathologic examination of the primary tumor, lymph node involvement, or distant metastases. ATCs were classified into three types: Common type, incidental type and anaplastic transformation type. In this study, we investigated only common type ATCs, and excluded incidental type and anaplastic transformation type.

Preoperative staging. Preoperative staging of patients included laryngoscopy, neck ultrasonography and computed tomography (CT) of head, neck and thorax to determine the extent of tumor infiltration. Only patients with a confirmed histopathological diagnosis of ATC by the surgical specimen were included. Poorly differentiated thyroid carcinomas were excluded. Patients were allocated to surgery, radiotherapy, chemotherapy, a combination of treatment modalities, or to best supportive care based on the preoperative staging.

Prognostic factors. Follow-up time started from the date of initial diagnosis, beginning in January 1990, and ended at either death, or December 2019. The primary endpoint of the study was the disease-related OS. To investigate prognostic 
factors for ATC, we compared survival rates by age, tumor size, WBC count, presence of distant metastasis, treatment by radical resection and tracheostomy.

Statistical analysis. Statistical analysis was performed with StatMate V statistical software (ATMS Co., Ltd.). Overall survival after ATC diagnosis was analyzed using the Kaplan-Meier method, and groups were compared using the log-lank test. P-values $<0.05$ were considered statistically significant. A multivariable analysis was not performed, due to the small number of the patients.

\section{Results}

Clinical characteristics. The clinical characteristics of the 17 evaluated patients are shown in Table I. Nine female and 8 male patients ranging from 58 to 84 years of age were included, with a median age of 74 years.

Treatment strategies. The treatment strategies are shown in Table II. Six of 17 patients with IVA/B carcinomas underwent potentially curative treatments. Two patients received radical resection, three patients radical resection with radiotherapy and one patient radical resection with chemotherapy. Four patients received radiotherapy only. Only one of 17 patients received best support care. In five of 17 patients, tracheostomies were performed.

Prognostic factors. Median OS of all patients was 3.8 months (range 6-442 days). Survival rates at 6 and 12 months after the initial diagnosis were 17.6 and $5.9 \%$, respectively (Fig. 1). The median OS of patients under the age of 70 , without distant metastasis and who had radical resection, were significantly longer compared to those patients over the age of 70 , with distant metastasis and no treatment by radical resection $(\mathrm{P}=0.010, \mathrm{P}=0.00022$ and $\mathrm{P}=0.0036$, respectively; Figs. 2-4) There was no significant effect on OS from tumor size or WBC count. Moreover, tracheostomy did not contribute to improvement of prognosis $(\mathrm{P}=0.71)$. The prognostic factors of ATC in this study are shown in Table III.

\section{Discussion}

ATC accounts for $1-2 \%$ of all thyroid carcinomas and is characteristic for fast progression, local invasion and a high rate of distant metastasis, all which result in poor prognosis (8). A generally accepted treatment strategy for ATC is not yet established $(9,10)$. Despite prolonged overall survival obtained by a combination therapy consisting of surgery, radiotherapy and chemotherapy (median survival of 9.9 months), more clinical research is still needed for the treatment of ATC (11). Radical treatment may worsen the quality of life, and occasionally even shorten the survival. Selecting patients who will benefit from either aggressive therapy or only supportive care is important in the management of ATC.

In 2001, Sugitani et al (12) devised a prognostic index (PI) based on four unfavorable prognostic factors present in patients with ATC. The factors were, i) acute symptoms (duration of severe complaints such as dysphonia, dysphagia, dyspnea and rapid growth of the tumor $<1$ month); ii) WBC
Table I. Characteristics of patients with anaplastic thyroid cancer.

\begin{tabular}{lc}
\hline Characteristics & Value \\
\hline Number of patients & 17 \\
Sex, $\mathrm{n}$ & \\
Female & 9 \\
Male & 8 \\
Age, years (range) & $74(58-84)$ \\
Median tumor size, mm (range) & $64(38-100)$ \\
Median white blood cell count, & $10,800(3,800-26,300)$ \\
mm ${ }^{2}$ (range) & \\
Distant metastasis, $\mathrm{n}$ & 5 \\
Radical resection, $\mathrm{n}$ & 6 \\
Tracheostomy, $\mathrm{n}$ & 5 \\
\hline
\end{tabular}

Table II. Performed treatment in patients with anaplastic thyroid cancer.

Potentially curative treatment No. of patients $(\%)$

\begin{tabular}{ll}
\hline Radical resection & $6(35)$ \\
Radical resection only & $2(12)$ \\
Radical resection + RT & $3(18)$ \\
Radical resection + PTX & $1(6)$ \\
RT & $4(24)$ \\
CT & $6(35)$ \\
Best supportive care & $1(6)$
\end{tabular}

RT, radiotherapy; PTX, paclitaxel; CT, chemotherapy.

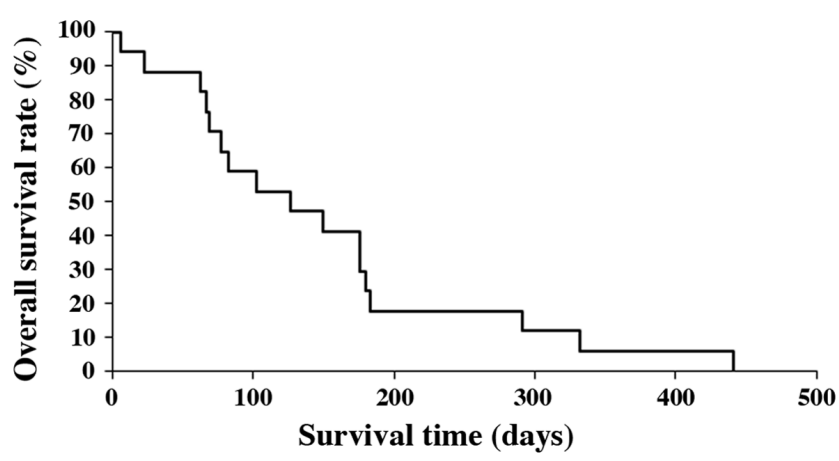

Figure 1. Kaplan-Meier analysis of overall survival of all 17 cases.

count $>10,000 / \mathrm{mm}^{2}$; iii) tumor size $>5 \mathrm{~cm}$, and iv) distant metastasis. In the present study, patients with $\mathrm{PI}=1$ showed a $62 \%$ survival rate at 6 months, whereas no patients with PI=3 or 4 survived longer than 6 months. All patients with PI=4 died within 3 months. Moreover, according to the ATC Research Consortium of Japan (ATCCJ) established in January 2009, that in addition to PI, both aged over 70 and T4b-stage tumor were confirmed to be significant risk factors for cause-specific death from ATC (3). 


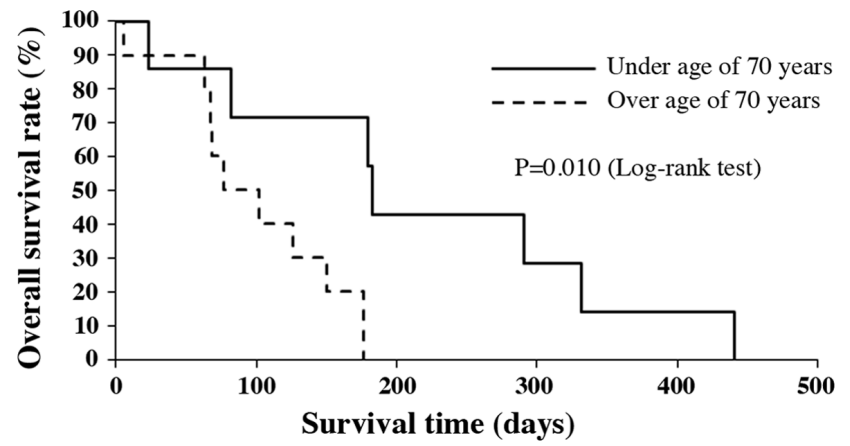

Figure 2. Kaplan-Meier analysis of overall survival by age.

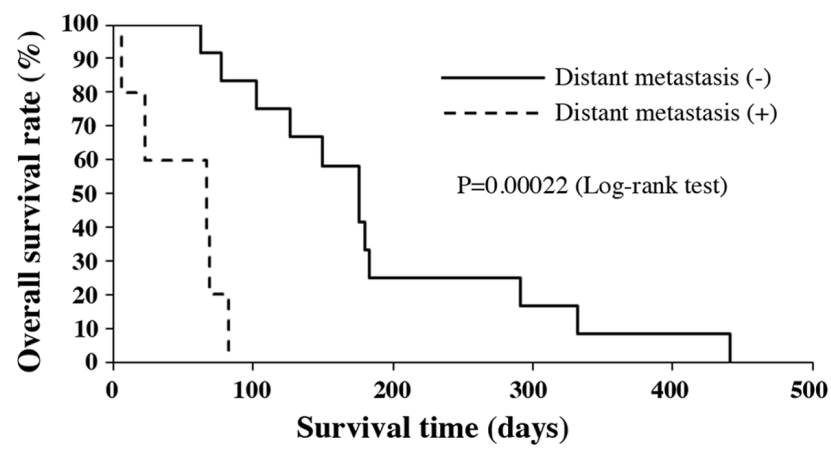

Figure 3. Kaplan-Meier analysis of overall survival by distant metastasis status.

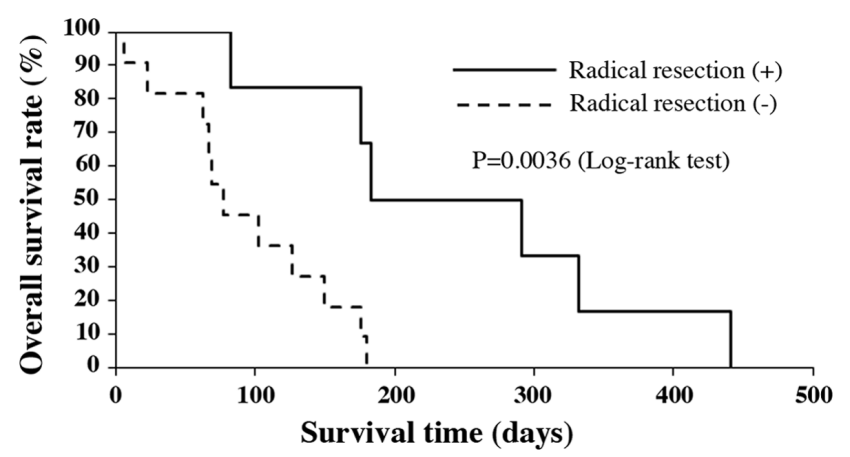

Figure 4. Kaplan-Meier analysis of overall survival by radical resection status.

We found the median OS of all patients was 3.8 months with 6 and 12 months survival rates of 17.6 and 5.9\%, respectively, in agreement with previous reports. Median OS was significantly longer in patients under the age of 70 , without distant metastasis, and who had radical resection. These results also confirm the opinion of the ATCCJ. In contrast, we found no significant differences regarding tumor size and WBC count, even though they have previously been identified as prognostic factors and included in the prognostic index for ATC. This may be due to an insufficient number of cases in this study. Regarding T4b-stage tumors and acute symptoms, we were unable to thoroughly investigate these 17 cases. Moreover, tracheostomy did not improve prognosis, thus we recommend that it should be avoided, whenever possible, to preserve the quality of life of ATC patients.
Table III. Prognostic factors of anaplastic thyroid cancer.

\begin{tabular}{lc}
\hline Factors & P-value \\
\hline Age $(\geq 70$ vs. $<70$ years) & 0.01000 \\
Tumor size ( $\geq 50$ vs. $<50 \mathrm{~mm})$ & 0.38000 \\
White blood cell count $\left(\geq 10,000\right.$ vs. $\left.<10,000 \mathrm{~mm}^{2}\right)$ & 0.12000 \\
Distant metastasis ( + or -$)$ & 0.00022 \\
Radical resection $(+$ or -$)$ & 0.00360 \\
Tracheostomy (+ or -$)$ & 0.71000
\end{tabular}

P-values were obtained using the log-rank test. $\mathrm{P}<0.05$ was considered to indicate a statistically significant difference.

Clinicians should take both the Union for International Cancer Control (UICC) stage as an indication of disease extent, and other prognostic factors representing the grade of biological malignancy, into consideration when determining treatment and prognosis. A distant metastasis exceedingly worsens the prognosis of ATC patients.

\section{Acknowledgements}

Not applicable.

\section{Funding}

No funding was received.

\section{Availability of data and materials}

The datasets used and/or analyzed during the current study are available from the corresponding author on reasonable request.

\section{Authors' contributions}

TM, HU, IO and TKit conceived and designed the study. TM, HU, IO, TKim, DN, TY, KY and TKit acquired data. TM, HU, IO, TKim and DN analyzed and interpreted data. TM and TKit wrote the original draft of the manuscript. TM, HU, IO and TKit reviewed and edited the manuscript. All authors have accepted their responsibility for the entire content of this manuscript and approved the submission. All authors read and approved the final manuscript.

\section{Ethics approval and consent to participate}

The present study was approved by Ethics Committee of Nara Medical University Hospital, and written informed consent was obtained from the patients.

\section{Patient consent for publication}

Not applicable.

\section{Competing interests}

The authors declare that they have no competing interests. 


\section{References}

1. Wachter S, Vorlander C, Schabram J, Mintziras I, Fulber I, Manoharan J, Holzer K, Bartsch DK and Maurer E: Anaplastic thyroid carcinoma: Changing trends of treatment strategies and associated overall survival. Eur Arch Otorhinolaryngol 277: 1507-1514, 2020

2. Shinohara S, Kikuchi M, Naito Y, Fujiwara K, Hori S, Tona Y, Yamazaki H, Kobayashi H and Ishihara T: Successful treatment of locally advanced anaplastic thyroid carcinoma by chemotherapy and hyperfractionated radiotherapy. Auris Nasus Larynx 36: 729-732, 2009.

3. Sugitani I, Onoda N, Ito KI and Suzuki S: Management of anaplastic carcinoma: The fruits from the ATC research consortium of Japan. J Nippon Med Sch 85: 18-27, 2018.

4. Cabanillas ME, Zafereo M, Gunn GB and Ferrarotto R: Anaplastic thyroid carcinoma: Treatment in the age of molecular targeted therapy. J Oncol Pract 12: 511-518, 2016.

5. Sykorova V, Dvorakova S, Vcelak J, Vaclavikova E, Halkova T, Kodetova D, Lastuvka P, Betka J, Vlcek P, Reboun M, et al: Search for new genetic biomarkers in poorly differentiated and anaplastic thyroid carcinoma using next generation sequencing. Anticancer Res 35: 2029-2036, 2015.
6. Kwon J, Kim BH, Jung HW, Besic N, Sugitani I and Wu HG: The prognostic impacts of postoperative radiotherapy in the patients with resected anaplastic thyroid carcinoma: A systematic review and meta-analysis. Eur J Cancer 59: 34-45, 2016.

7. Pierie JP, Muzikansky A, Gaz RD, Faquin WC and Ott MJ: The effect of surgery and radiotherapy on outcome of anaplastic thyroid carcinoma. Ann Surg Oncol 9: 57-64, 2002.

8. Kebebew E, Greenspan FS, Clark OH, Woeber KA and McMillan A: Anaplastic thyroid carcinoma. Treatment outcome and prognostic factors. Cancer 103: 1330-1335, 2005.

9. Granata R, Locati L and Licitra L: Therapeutic strategies in the management of patients with metastatic anaplastic thyroid cancer: Review of the current literature. Curr Opin Oncol 25: 224-228, 2013.

10. Smallridge RC, Marlow LA and Copland JA: Anaplastic thyroid cancer: Molecular pathogenesis and emerging therapies. Endocr Relat Cancer 16: 17-44, 2009.

11. Haymart MR, Banerjee M, Yin H, Worden F and Griggs JJ: Marginal treatment benefit in anaplastic thyroid cancer. Cancer 119: 3133-3139, 2013.

12. Sugitani I, Kasai N, Fujimoto Y and Yanagisawa A: Prognostic factors and therapeutic strategy for anaplastic carcinoma of thyroid. World J Surg 25: 617-622, 2001. 